\title{
Target therapy of multiple myeloma by PTX-NPs and ABCG 2 antibody in a mouse xenograft model
}

\author{
Cuiping Yang ${ }^{1, *}$, Fei Xiong ${ }^{2, *}$, Jun Dou ${ }^{1, *}$, Jun Xue ${ }^{3}$, Xi Zhan ${ }^{4}$, Fangfang Shi ${ }^{5}$, Miao Li ${ }^{1}$, \\ Songyan Wu ${ }^{1}$, Shouhua Luo $^{2}$, Tianzhu Zhang ${ }^{2}$, Yu Zhang ${ }^{2}$, Ji Ming ${ }^{2}$, Ning Gu ${ }^{2}$ \\ ${ }^{1}$ Department of Pathogenic Biology and Immunology, School of Medicine \& Collaborative Innovation Center of Suzhou \\ NanoScience and Technology, Southeast University, Nanjing 210009, China \\ ${ }^{2}$ School of Biological Science \& Medical Engineering \& Collaborative Innovation Center of Suzhou NanoScience and Technology, \\ Southeast University, Nanjing 210096, China \\ ${ }^{3}$ Department of Hematology, Affiliated Nanjing First Hospital, Nanjing Medical University, Nanjing 210006, China \\ ${ }^{4}$ The Center for Vascular and Inflammatory Diseases, Department of Pathology, University of Maryland School of Medicine, \\ Baltimore, MD 21201, USA \\ ${ }^{5}$ Department of Oncology, Zhongda Hospital, Southeast University, Nanjing 210009, China \\ *These authors have contributed equally to this work \\ Correspondence to: \\ Jun Dou, e-mail: njdoujun@seu.edu.cn \\ Ning Gu, e-mail: guning@seu.edu.cn \\ Keywords: multiple myeloma, cancer stem cells, paclitaxel, nanoparticles, ATP-binding cassette sub-family G member 2 \\ Received: May 01, 2015 \\ Accepted: July 06, 2015 \\ Published: July 15, 2015
}

\section{ABSTRACT}

Multiple myeloma (MM) remains to be an incurable disease. The purpose of this study was to evaluate the effect of ABCG2 monoclonal antibody (McAb) combined with paclitaxel (PTX) conjugated with $\mathrm{Fe}_{3} \mathrm{O}_{4}$ nanoparticles (NPs) on MM progressed from cancer stem cells (CSCs)in non-obese-diabetic/severe-combinedimmunodeficiency (NOD/SCID) mouse model. Mice were injected with MM CSCs as marked by CD138-CD34- phenotypes through tail veins. The developed MM mice were examined by micro-computer tomography scanning, ultrasonography and enzymelinked immunosorbent analysis. These mice were then intravenously treated with different combinations of NPs, PTX, McAb, PTX-NPs and melphalan/prednisone once a week for four weeks. The injected mice developed characteristic MM-associated syndromes, including lytic bone lesions, renal damages and proteinuria. All the treated mice showed decrease in bone lesions, renal damages and anemia but increase in apoptosis compared with the mice treated with NPs only. In particular, the treatment with ABCG2 MCAb plus PTX-NPs induced the strongest therapeutic response and had an efficacy even better than that of melphalan/prednisone, a conventional regimen for MM patients. These data suggest that PTX-NPs with ABCG2 McAb can be developed into potential treatment regimens for patients with relapsed/refractory MM.

\section{INTRODUCTION}

Multiple myeloma (MM) is a debilitating disease characterized by neoplastic growth of plasma cells in the bone marrow and responsible for the most deaths of patients with hematologic malignancies. Clinically, MM patients often manifest destructive bone lesions, renal insufficiency, hypercalcemia and anemia. Agents of thalidomide, lenalidomide, bortezomib, melphalan/ prednisone, and carfilzomib as well as hematopoietic stem cell transplantation have dramatically improved the therapeutic response and survival rate of MM patients. However, most MM patients are still facing a high risk of ultimate relapses due to the persistence of minimal residual malignant cells in the bone marrow. Consequently MM remains to be an incurable disease [1,2]. Emerging evidence supports the view that a subpopulation of MM cells that are highly resistant to conventional cancer 
therapies shares certain features with cancer stem cells (CSCs) and may be responsible for tumor-initiating, selfrenewal, drug resistances and metastasis [3-6]. Thus, it is clinically desirable to develop a therapeutic paradigm that targets specific molecules associated with MM $\mathrm{CSCs}$, and thereby overcoming the drug resistance by eradicating CSCs.

It is known that CSCs often exhibit a high activity of ATP-binding cassette (ABC) transporter, in particular ABCG2 (ATP-binding cassette sub-family G member 2), a surface molecule that contributes to drug resistance by pumping out intracellular drugs. Indeed, there have been reports that using anti-ABC monoclonal antibodies are able to inhibit partially cellular drug resistance by compromising the growth of CSCs $[7,8]$.

Paclitaxel (PTX) has a broad spectrum of cytotoxicity towards a variety of cancers, including MM. However, it has a poor solubility, and clinical usage of its adjuvants such as Cremophor EL often causes severe allergic reactions, which significantly limits the efficacy of PTX-based therapies [6,9]. Recent advances have evidenced that engineered nanoparticles (NPs) offer many therapeutic advances over traditional formulations because they can be readily manipulated into particles with desired sizes and surface properties that would make it possible to conjugate and deliver certain pharmaceutical compounds that are otherwise insoluble and unstable [10-15]. Magnetic nanoparticles (NPs) such as magnetite $\mathrm{Fe}_{3} \mathrm{O}_{4}$ based NPs have small sizes, large active surface areas, an excellent biocompatibility, and are readily internalized into cells and sustain well under endocytic environments. In the presented study we attempted to target MM CSCs by using a monoclonal antibody against ABCG2, and to deliver PTX by using NPs. To evaluate the anti-MM efficacy of the new approach, we also developed a MM model in which MM CSCs derived from either human MM RPMI 8266 cells or bone marrow mononuclear cells (BMMCs) from MM patients were used to develop MM-like malgiancies in xonografted mice. We found that application of NPs carrying PTX in combination with the ABCG2 antibody improved significantly the efficacy of inhibiting MM progression compared with that using each agent alone.

\section{RESULTS}

\section{Mice injected with MM CSCs manifests typical MM-associated syndromes}

To establish a MM animal model, we isolated MM CSCs from human MM RPMI 8226 cells based on the CD138 ${ }^{-} \mathrm{CD}^{2} 4^{-}$cell phenotypes ${ }^{17}$ and injected them via tail veins into NOD/SCID mice. Because MM patients were frequently associated with bone lesions and kidney damages, we examined on day 18 after implantation the bone marrow density (BMD) of humeri and femora of injected mice by micro-CT, a gold standard for imaging MM [16]. As shown in Fig. 1A and 1B, the BMD of both humerus and femurs of MM CSC-injected mice (model) was significantly reduced compared with the control group (normal). Micro-CT also detected a few lowdensity shadows (arrows, Fig. 1C) in the kidney of model mice, suggestive of the presence of MM lesions and renal impairment. Indeed, ultrasound imaging analysis of the renal arteries revealed that the peak systolic blood flow velocity (BFV) in the renal artery was significantly decreased in model mice (Fig. 1D and 1E). To assess further the renal damage, we measured protein contents in the urine and found that the proteinuria level was markedly enhanced in the model mice versus the normal mice (Fig. 1F). Thus, mice injected with MM CSCs shared many pathological characteristics with those of MM.

\section{PTX-NPs combined with ABCG2 McAb reduces effectively MM-associated bone lesions}

To determine whether prepared PTX-NPs as illustrated in Fig. 2A remain a nano property, we analyzed PTX-NPs by transmission electron microscopy (TEM) and dynamic light scattering (DLS), which demonstrated that PTX-NPs have a quite uniformed core size of $7.63 \mathrm{~nm}$ (Fig. 2B), and hydrodynamic diameter of $69.0 \mathrm{~nm}$ (Fig. 2C), the latter of which includes both the core-shell and the aqueous layer.

To evaluate the therapeutic effect of PTX-NPs on MM progression, the mice injected with MM CSCs were treated once a week with different combinations of PTX, $\mathrm{NPs}$ and McAb. In addition, injected mice were treated with MP, which is a common and effective regimen for MM patients. After 4 weeks of treatments, the mice were subjected to BMD examination. As shown in Fig. 3A, the lowest BMD was found with the model group in which the injected mice were treated with PBS only. The group of which the injected mice were treated with NPs alone also showed a poor BMD. In contrast, the mice treated with the other combinations showed improved BMD at different degrees. Significantly, the mice that were treated with McAb-PTX-NPs had restored BMD up to the level similar to that of the normal group and even higher than that of mice treated with MP (Fig. 3B).

Bone lesions in treated mice were further analyzed by histology. The model mice had developed significant lytic bone lesions, including bone trabeculae destruction, sinus extension and breakage, RBC seepage and edema in the bone marrow matrix, and aggregated and infiltrated inflammatory cells (arrows) in the compact bone (Fig. 4A). The lytic bone lesion, as determined by infiltrated inflammatory cells, was markedly decreased in the mice treated with McAb-PTX-NPs compared with 
A

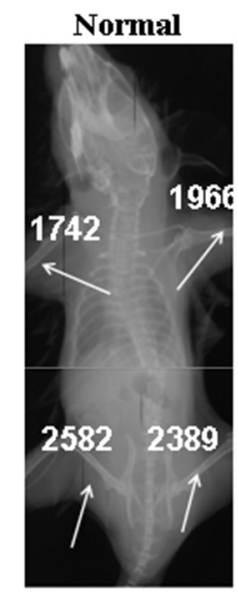

D

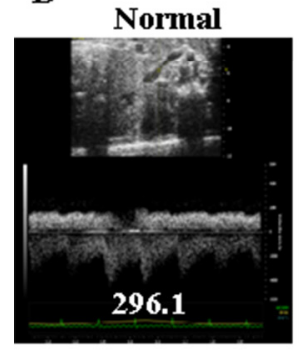

B

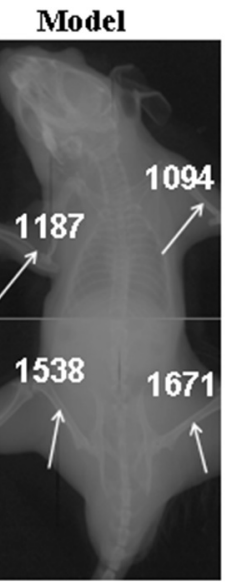

Model

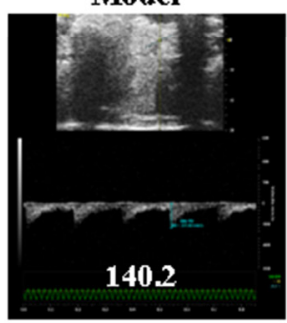

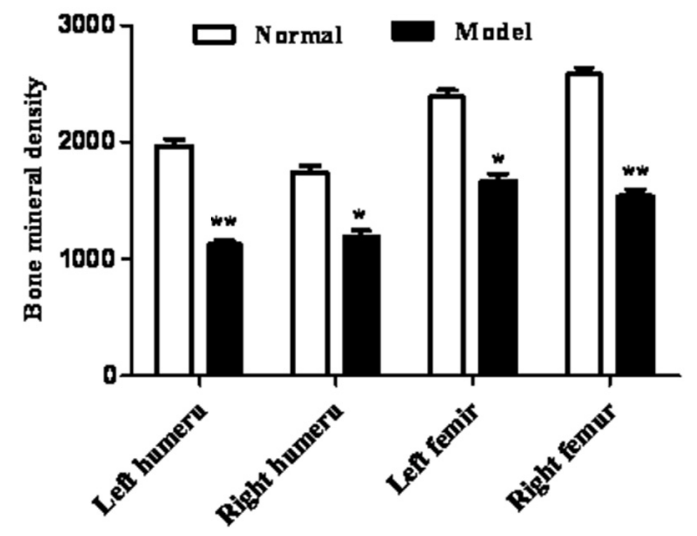

E

F

C
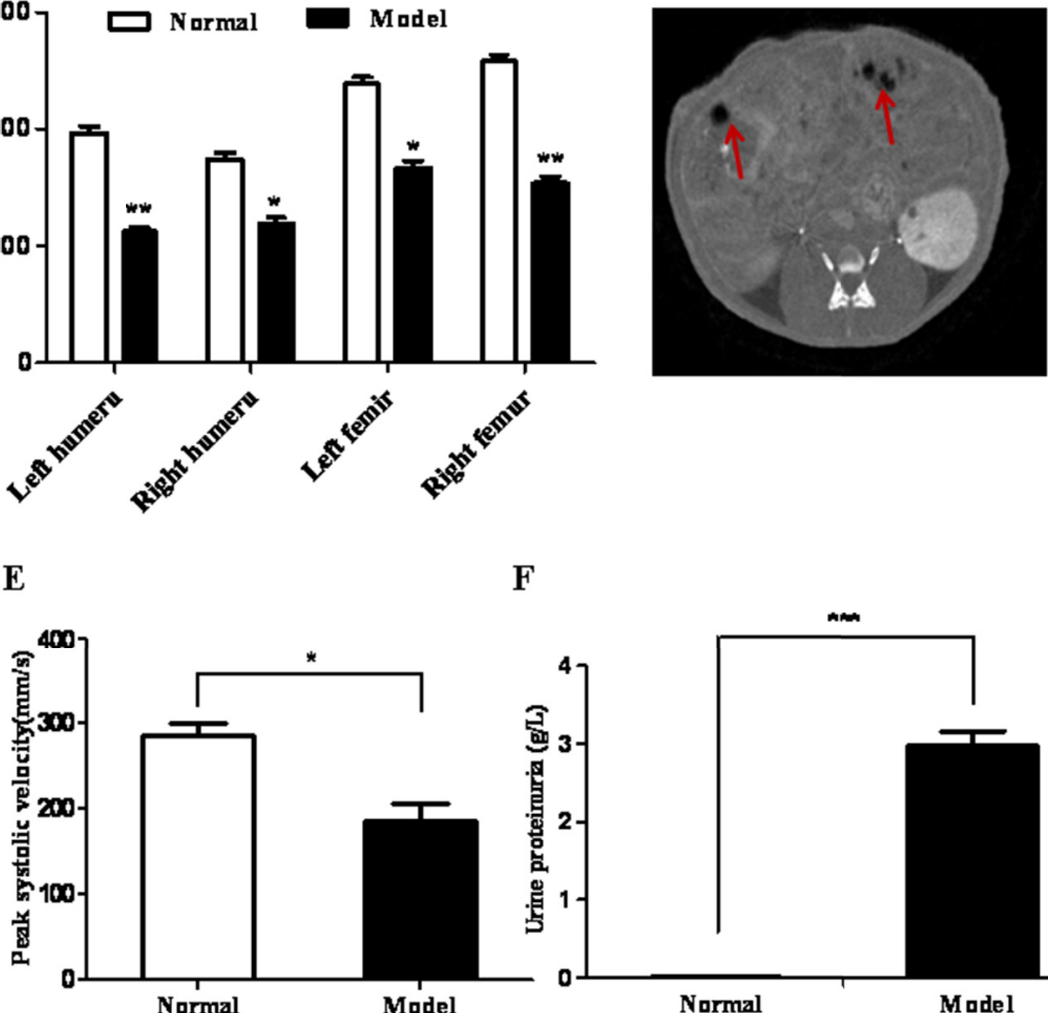

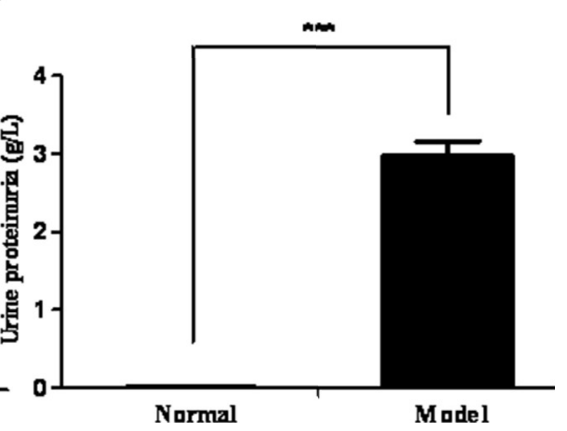

Figure 1: Establishment of a MM murine model. A. Images showed Micro-CT scanning of a normal and a model mouse 18 days after injection of MM CD138 CD34 cells. BMD values at femur and humerus were indicated by arrows. B. Quantification of BMD as measured by Micro-CT $(n=6)$. C. A Micro-CT image of kidneys of a model mouse. Arrows indicate renal damaged areas. D. Ultrasound images showing peak systolic BFV of the renal artery of a normal and a model mouse. E. Quantification of peak systolic BFV. F. Urine protein was measured by ELISA. All the data represent mean \pm S.D $(n=3) .{ }^{*} p<0.05,{ }^{* *} p<0.01$ and $* * * p<0.001$ were calculated by $t$ test, referring to the statistically significant difference as compared to the normal group.

those treated with McAb, PTX, PTX-NPs, and model mice, respectively. However, there was no significant difference between the McAb-PTX-NP group and the MP group (Fig. 4B).

\section{McAb-PTX-NPs improves the kidney function of MM bearing mice}

To evaluate whether McAb-PTX-NPs have any therapeutic effect on the MM-mediated kidney disorders, we analyzed the renal artery of treated mice by ultrasound imaging. As shown in Fig. 5, the renal artery of normal mice had a stable current velocity profile and a peak $\mathrm{BFV}$ at 295.9, whereas that of MM bearing mice without treatment had a nearly diminished current velocity wave and markedly decreased BFV (140.1). Notably, the peak BFV of the renal artery of the mice treated with McAbPTX-NPs was significantly recovered to 254.6, which was accompanied with a stable arterial current velocity. The mice treated with MP also showed a significant recovery and had a peak BFV at 250.5, which was statistically the same as that treated with McAb-PTX-NPs. However, these mice had a less normal current velocity profile than did McAb-PTX-NPs treated mice.

Renal sections derived from the mice after 28 days of treatments were also subjected to histological analysis. Apparent renal damages as indicated by large blue areas were seen in massion-stained sections from the model mice (Fig. 6A, top panel). Also, periodic acid-schiff staining revealed that the model mice had over $75 \%$ glomeruli that were apparently hardened (bottom panel), which is pathologically equivalent to a 4 score of renal damages $[15,17]$. The renal damages became apparently less severe in the mice treated with the other agents. In particular, the mice treated with McAb-PTX-NPs or MP showed a significant alleviation of renal damages (Fig. 6B).

Kidney damages in MM patients are often manifested by increase in protein contents in urine and in the levels of serum free light chains (FLCs), calcium and IL-6 in serum [17-20]. To analyze the effect of McAbPTX-NPs on these MM-associated disorders, we used ELISA to measure the contents of these soluble factors. The result of this study indicated that the treatment of MM 
A

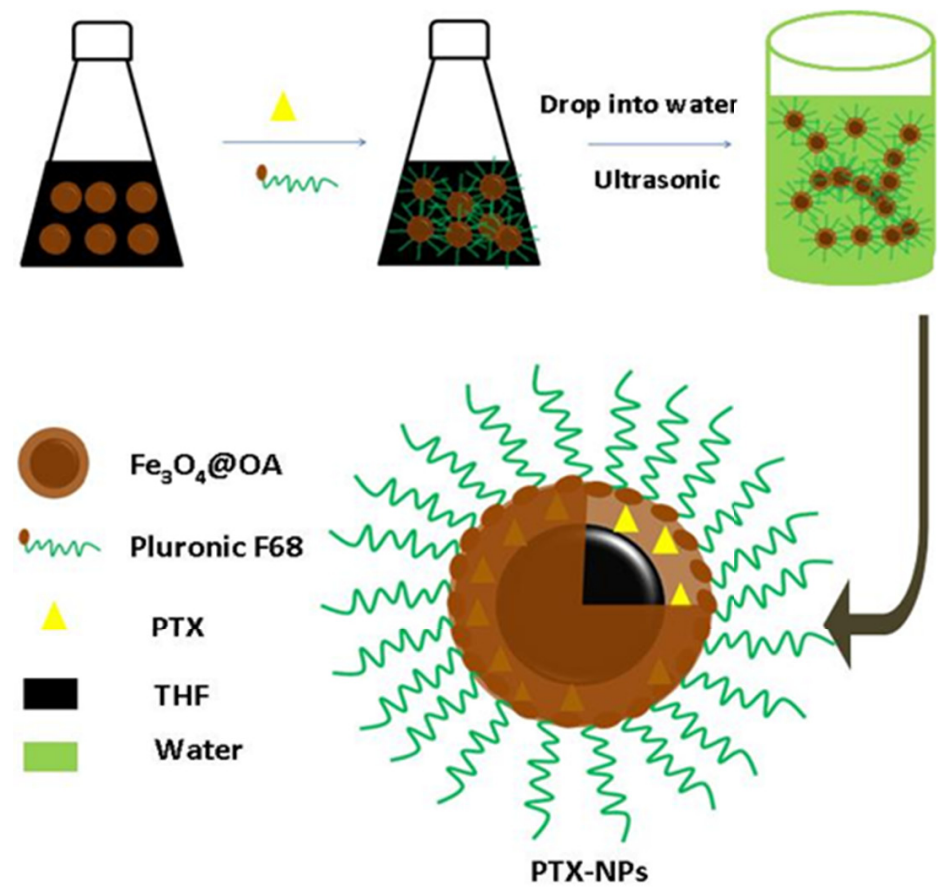

B

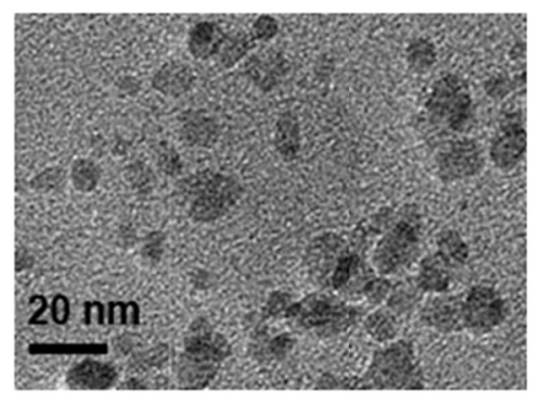

TEM: Average size $7.63 \mathrm{~nm}$

$\mathrm{C}$

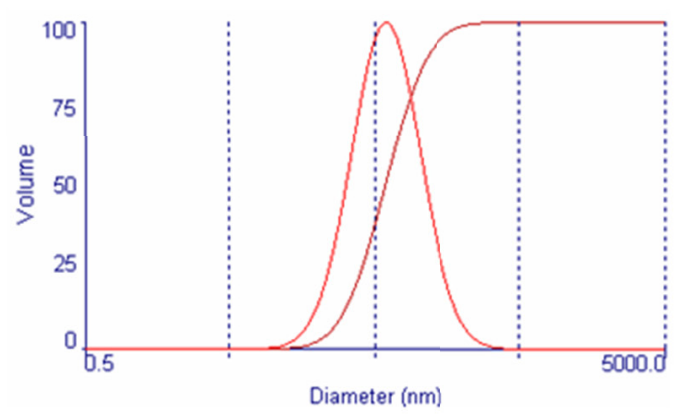

DLS: Mean Diameter $69.0 \mathrm{~nm}$

Figure 2: Characterization of PTX-NPs. A. Schematic illustration of PTX-NPs preparation. PTX, oleic acid-coated iron oxide nanoparticles (Fe3O4@OA NPs) and polyoxyethylene/polyoxypropylene copolymer (Pluronic F68) were dissolved in tetrahydrofuran (THF). The mixture was added into water under sonication, and free THF in the resulting suspension was evaporated under a continuous stirring. B. A TEM image showing prepared nanoparticles conjugated with PTX. The average size of PTX conjugated nanoparticles is about $7.63 \mathrm{~nm}$ as estimated by an image analysis program based on more than 300 particles. C. The hydrodynamic diameter distribution of PTX-NPs was measured by dynamic light scattering (DLS).

mice with PTX-NPs significantly decreased the levels of FLC, calcium and IL-6 in serum as well as proteins in urine compared with that of PTX or McAb alone (Fig. S1). This efficiency was further enhanced by combining PTXNPs with ABCG2 McAb. In addition to the soluble molecules, the number of RBCs, as measured by RBC counting, was also elevated similarly in the mice treated by these agents (Fig. S1).

\section{McAb-PTX-NPs induces strong apoptosis with BMMCs derived from MM mice}

To explore a cellular mechanism for the anti-MM function of McAb-PTX-NPs, we performed flow cytometry to analyze the apoptosis with BMMCs derived from treated mice. As shown in Fig. 7A, normal and model mice or the mice treated with NPs contained few apoptotic BMMCs. In contrast, mice treated with other agents showed a marked increase in the number of apoptotic BMMCs. Among them, McAb-PTX-NPs caused the highest apoptotic rate $(58.98 \%)$, which is significantly greater than those induced by PTX, or McAb or PTX-NPs alone, which ranged from $21.02 \%$ to $38.83 \%$ (Fig. $7 \mathrm{~B}$ ).

\section{McAb-PTX-NPs has a strong therapeutic effect on cancer cells derived from MM patients}

To determine whether McAb-PTX-NPs have a therapeutic effect on primary MM cells, we isolated CD138 ${ }^{-} \mathrm{CD}^{-} 4^{-}$cells from BMMCs of three MM patients. These cells have characteristics of CSCs as determined by a series of in vitro assays (Fig. S2), which demonstrated that $\mathrm{CD} 138^{-} \mathrm{CD} 34^{-}$cells had a higher growth rate (Fig. S2A), a higher clonogenic potential (Fig. S2B and $2 \mathrm{C}$ ), a higher drug resistance to vincristine (Fig. S2D), and a higher ability to form tumors in xenografted mice than did non-CD138 ${ }^{-}$CD $34^{-}$cells (Fig. S2E). In addition, CD138- ${ }^{-}$D34 ${ }^{-}$cells from nine MM patients showed a significantly increased expression levels of ABCG2 at both mRNA and cell surface (Fig. S3).

Upon treatment with McAb-PTX-NPs, MM-derived $\mathrm{CD} 138^{-} \mathrm{CD} 34^{-}$cells underwent to significant apoptosis 
A
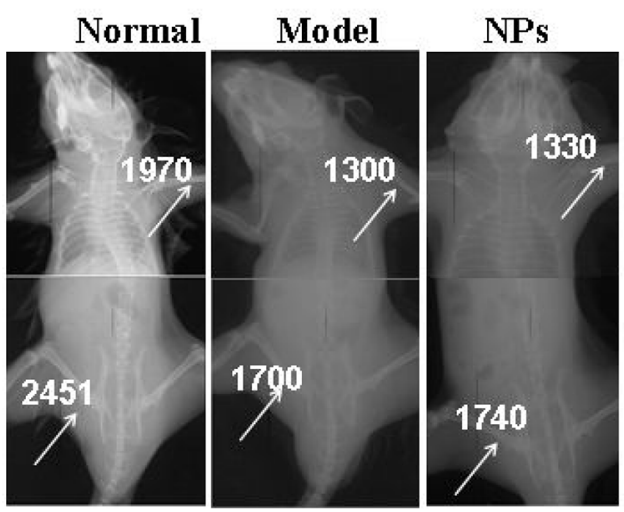

McAb

MP

PTX
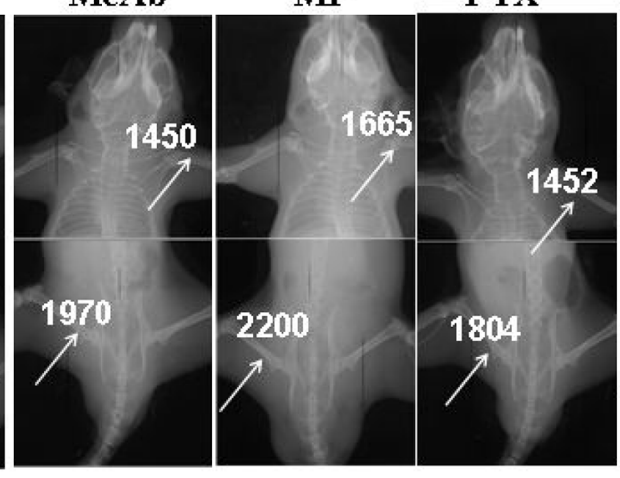

PTX-NPs McAb+PTX-NPs
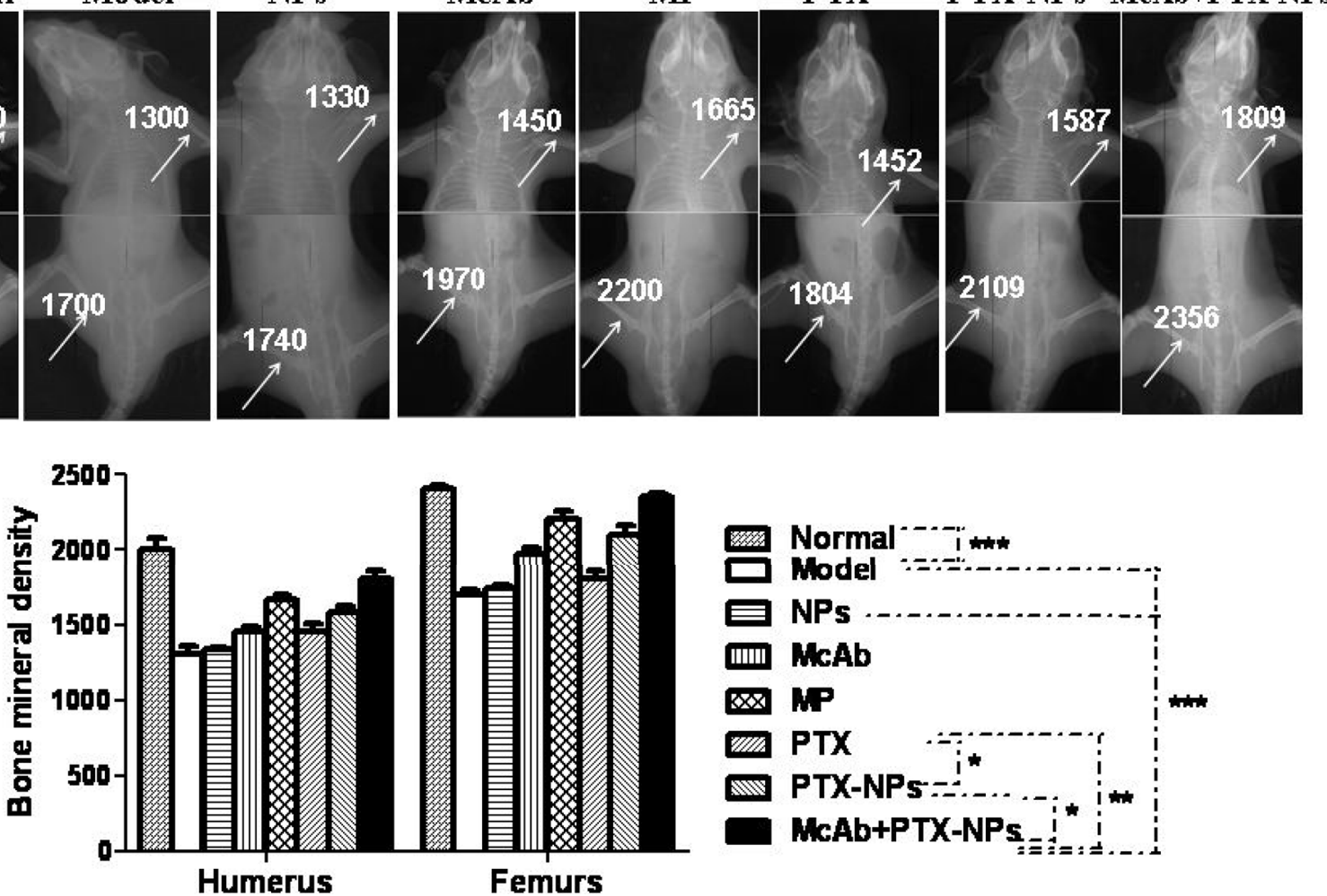

B

Humerus

Femurs

Figure 3: Significant improvement of BMD by McAb+PTX-NPs in MM mice. A. Micro-CT images showing BMDs in MM mice 4 weeks after treatments with different agents as indicated. B. Quantification of BMD in the mice treated with different agents. The data represent mean $\pm \mathrm{SD}(n=6) * p<0.05, * * p<0.01$ and $* * * p<0.001$ referred to the differences as indicated. Bonferroni correction was applied if multiple comparisons were involved.

in vitro (Fig. S4). Application of McAb-PTX-NPs to NOD-SCID mice injected with $5 \times 10^{4} \mathrm{CD} 138^{-} \mathrm{CD} 34^{-}$cells increased significantly their BMD compared with the control mice (data not shown), demonstrating that McAbPTX-NPs have also a strong therapeutic effect on primary MM cells.

\section{DISCUSSION}

MM CSCs may be responsible for chemoresistances, the primary cause for the clinical failure in complete elimination of MM cells. One of the possible mechanisms for drug resistance is that CSCs express high levels of ABCG2 transporter [8, 21, 22], which facilitates pumping out therapeutic drugs out of cells. Recent advances have evidenced that the targeted therapies have promised to improve the efficacy of cancer treatments by aiming at inhibition of specific molecules or signaling pathways. Thus, we hypothesized that combination of a conventional cancer drug with the ability to target ABCG2 would be a better approach to treat MM patients and may improve drug-sensitivity. In this study, we focused on $\mathrm{CD} 138^{-} \mathrm{CD} 34^{-}$cells because this phenotype cells isolated from MM cell line RPMI 8226 have the characteristics of MM CSCs, which exhibited stronger proliferation, migration, drug resistance to PTX, clone formation ability, tumorigenic potential and more ABCG2 molecular expression than the non-CD138-CD34-cells $[23,24]$. In addition, we found that such population derived from MM patients possesses typical CSC features and that they are enriched in ABCG2 expression on cell surface. Our finding is essentially consistent with the previous findings by others $[2,22,25]$ and by us $[23,24]$. Indeed, we had previously been able to establish tumors in mice by subcutaneous injection of $\mathrm{MM} \mathrm{CD} 138^{-} \mathrm{CD} 34^{-} \mathrm{CSCs}$ from human cell lines. However, whether these injected mice have MM characteristics remained unknown. In the presented study we injected, via dorsal tail vein, $\mathrm{MM}$ CD $138^{-} \mathrm{CD} 34^{-} \mathrm{CSCs}$ into NOD/SCID mice and demonstrated that these injected mice developed typical MM-associated symptoms including abnormally poor BMD, high levels of urine protein, high levels of FLC, calcium and IL-6, and impairments in the function of kidney. In view of the fact that mice normally do not contain detectable level of protein in urine and that the 

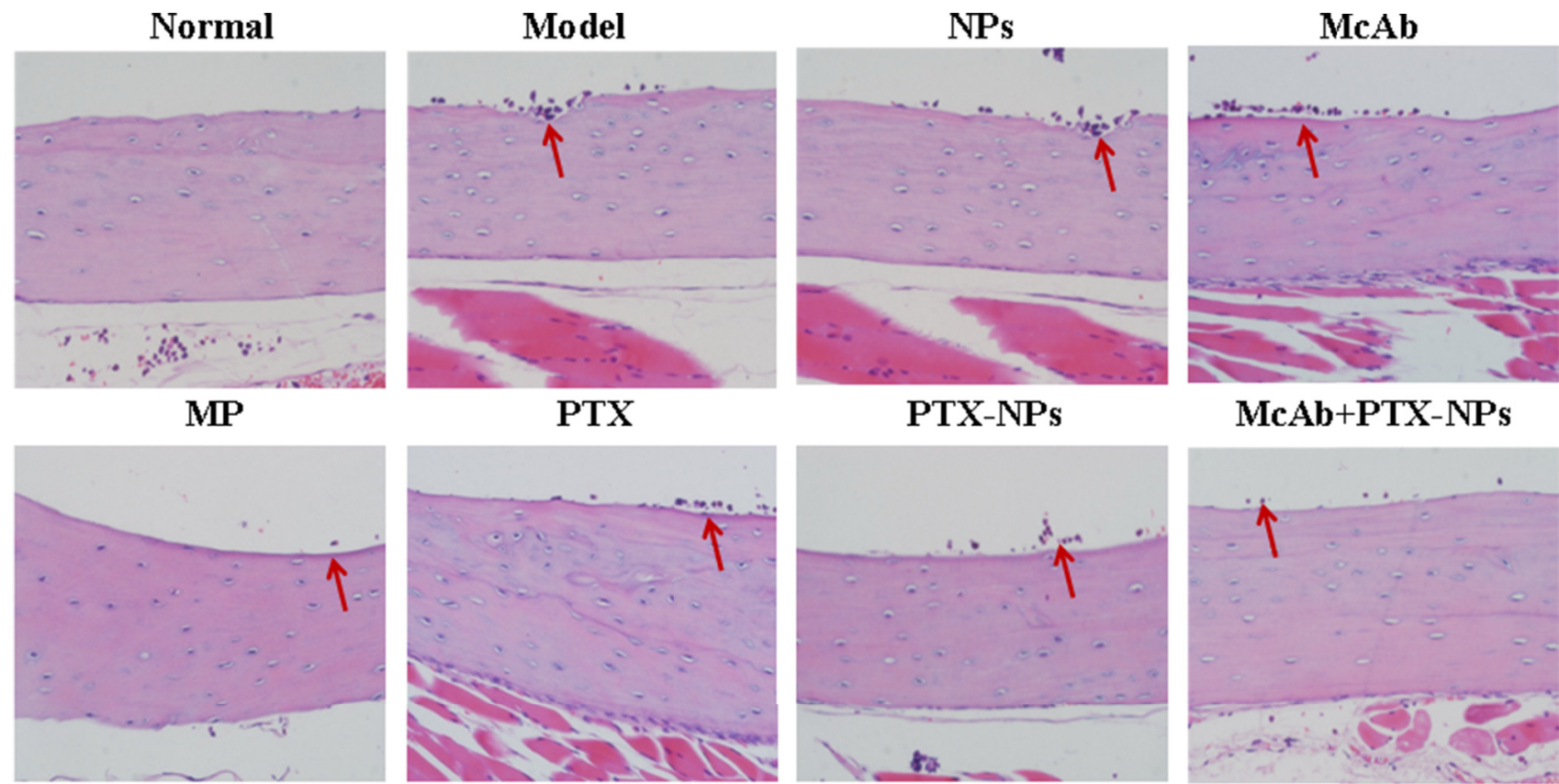

McAb+PTX-NPs

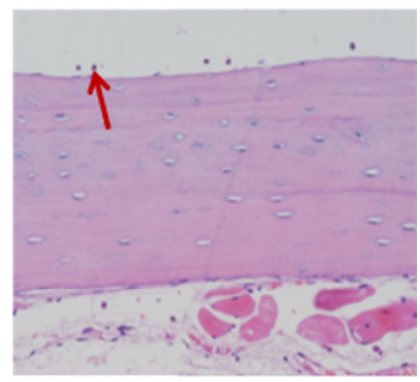

A
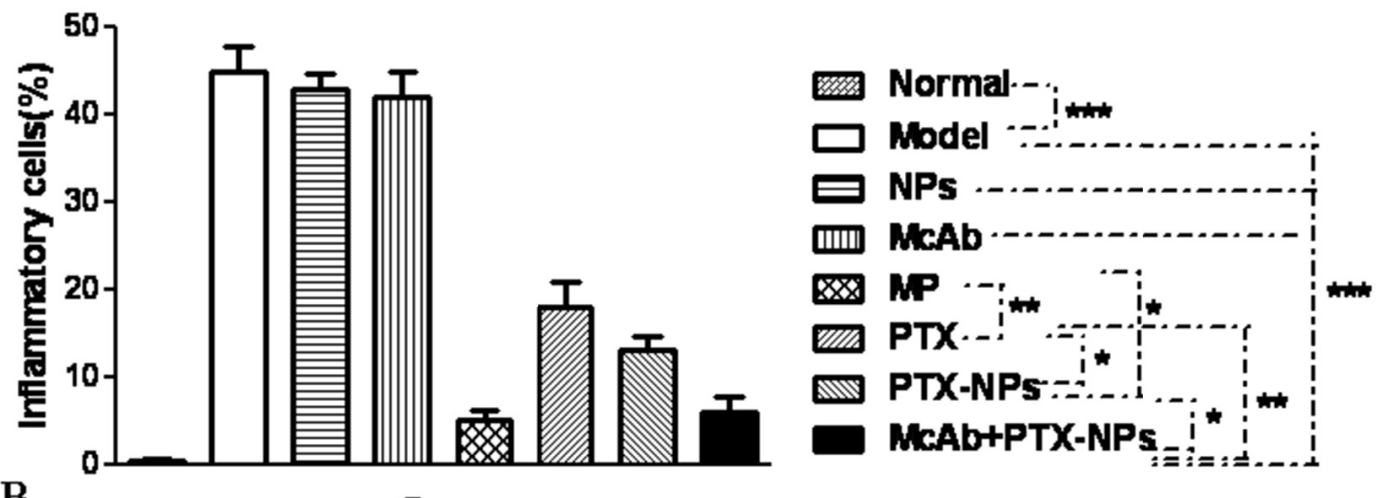

B

\section{Groups}

Figure 4: Bone lesion reduction by McAb+PTX-NPs in MM mice. A. HE staining of femur lesions in MM mice 4 weeks after treatment with different agents as indicated (magnification, $400 \times$ ). Arrows indicate aggregated and infiltrated inflammatory cells. B. Quantification of bone lesions. ${ }^{*} p<0.05,{ }^{*} p<0.01$ and $* * * p<0.001$, referring to the differences as indicated.

mice injected with CD138- CD34- ${ }^{-}$SCs exhibited a high level of urinal protein, it is likely that injected MM cells had significantly infiltrated into the kidney. Thus, the mice described here would serve as a proper murine model that reflect many pathological aspects associated with MM. Based on this model, we have been able to demonstrate that the combination of PTX-NPs with ABCG2 McAb achieved a highest therapeutic response than did any other combinations tested in this study. Importantly, McAb-PTX-NPs showed even a stronger efficacy than MP, which has a demonstrated curative effect on MM [26, 27]. Thus, combination of nano-drugs with ABCG2 McAb targeting CSCs could be ultimately developed into a better therapy for MM.

The strong therapuetic activity of McAb-PTXNPs is apparently associated with its strong activity to induce BMMC apoptosis. The mechanisms may involve inhibition of $\mathrm{ABCG} 2$ and preventing of the internalized
PTX from efflux out of the cells, and thereby generating a high cytotoxicity on cells. In addtion, we guess that antiABCG2 McAb may lyse MM cells by antibody-dependent cell-mediated toxicity and complement-dependent cytotoxicity in mice. These possible mechanisms deserve further investigations.

In conclusion, our data shows the targeted therapeutic effect of McAb-NPs-PTX on MM CD $138^{-} \mathrm{CD} 34^{-} \mathrm{CSC}$ s were demonstrated in a xenograft MM mice. To our knowledge, this study is the first to examine the efficacy of McAb-PTX-NPs therapy for MM murine model established by tail vein injection of MM $\mathrm{CD} 138^{-} \mathrm{CD}^{-} 4^{-}$cells. The findings presented provide novel insight into the use of NPs-PTX in combination with antiABCG2 McAb directly targeting MM CD $138^{-} \mathrm{CD} 34^{-} \mathrm{CSCs}$ and inhibiting CSC growth in vivo. The strategy may be an effective treatment paradigm for patients with relapsed/ refractory MM. 
$\mathbf{A}$

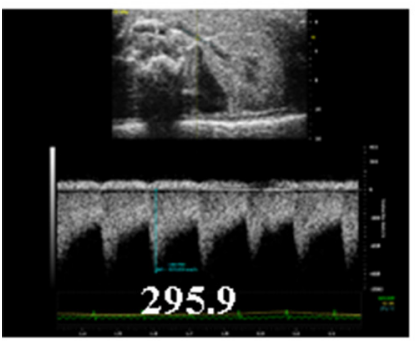

MP

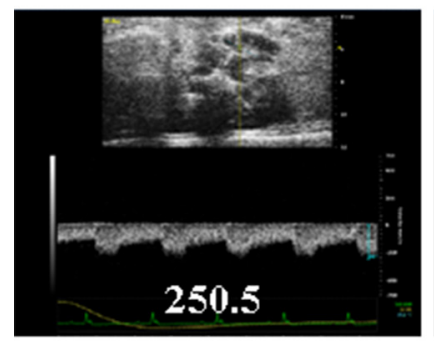

Model

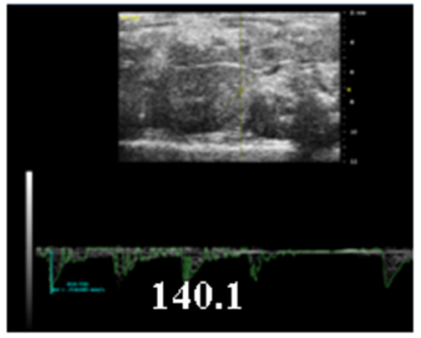

PTX

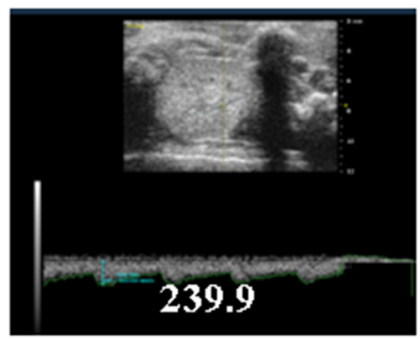

NPs

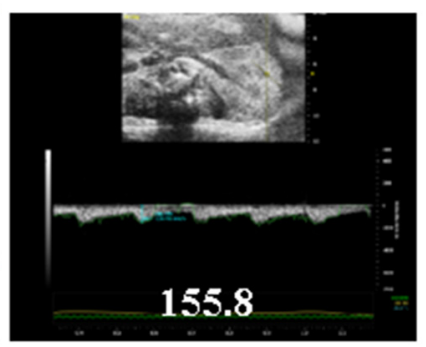

PTX-NPs

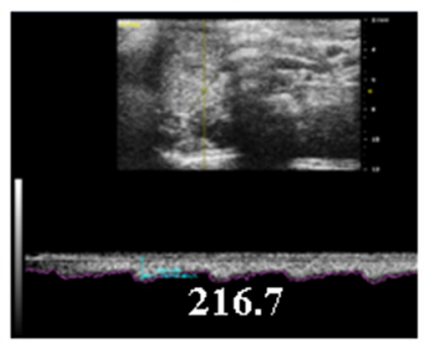

McAb

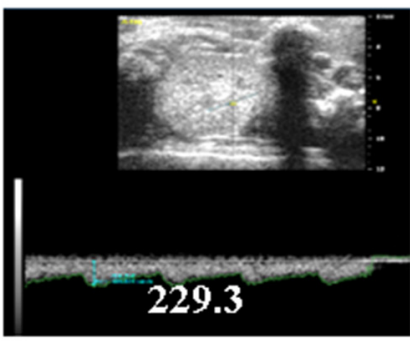

McAb+PTX-NPs

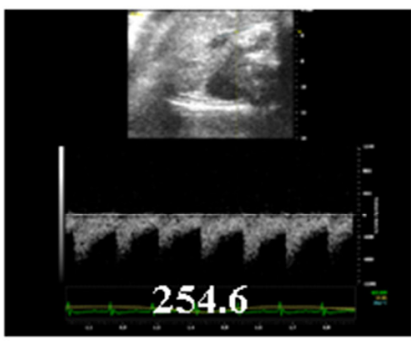

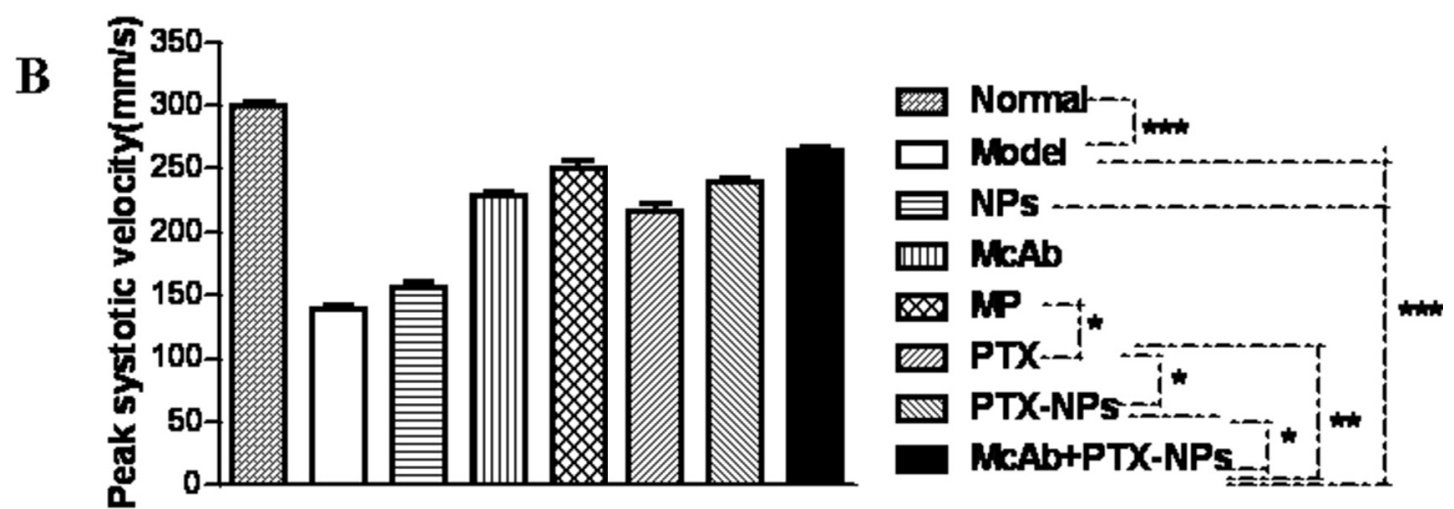

Groups

Figure 5: Ultrasound imaging of the BFV of renal arteries in MM mice. A. Images showing peak systolic BFV of renal arteries in MM mice 4 weeks after treatment with different agents as indicated. B. Statistical analysis of peak systolic BFV of MM mice. ${ }^{*} p<0.05$, $* * p<0.01$ and $* * * p<0.001$.

\section{MATERIALS AND METHODS}

\section{Cell line}

The human MM RPMI 8226 cell line was purchased from the Cellular Institute of Basic Medical Science, Chinese Academy of Medical Sciences in Bejing, China. Cells were cultured in complete media consisting of RPMI 1640, $2 \mathrm{mM}$ L-glutamine, $100 \mathrm{U} / \mathrm{mL}$ penicillin, $100 \mu \mathrm{g} / \mathrm{mL}$ streptomycin, and $10 \%$ fetal bovine serum at $37^{\circ} \mathrm{C}$ with $5 \% \mathrm{CO}_{2}$.

\section{Clinical samples}

Clinical bone marrow samples were obtained from $9 \mathrm{MM}$ patients at stages II-III with a median age of 65 years (55-86 years) or healthy donors who granted informed consent as approved by the Nanjing
First Hospital Affiliated to Nanjing Medical University. BMMCs from the MM patients were isolated by density centrifugation using Ficoll-Paque solution (Biological Chemical Factory, Shanghai, China).

\section{Animals}

Non-obese-diabetic/severe-combinedimmunodeficiency (NOD/SCID) mice at 5 weeks of age with $16 \pm 2$ gram in weight were purchased from Beijing Weitong Lihua Experimental Animal Technology Co., Ltd., China. All the mice were maintained in a pathogenfree facility that has a 12-hour light/dark cycle and relative humidity ranged from $40 \%$ to $50 \%$. The enviromental temperature was maintained at $22^{\circ} \mathrm{C}$ and the mice were fed with steril foods. All the animal experiments were performed in compliance with the Guidelines of the Animal Research Ethics Board of Southeast University. 


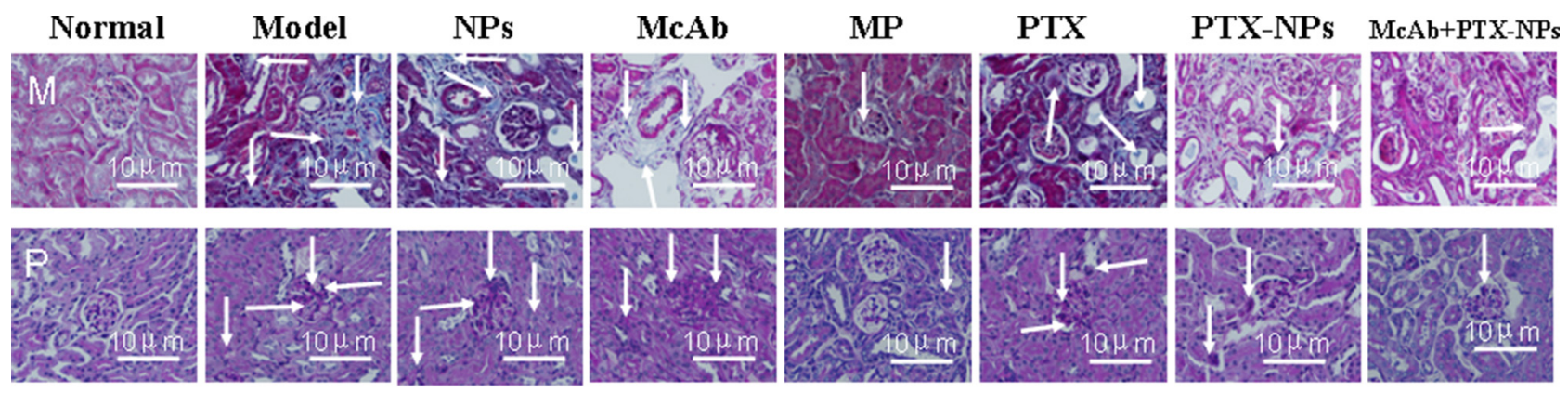

A

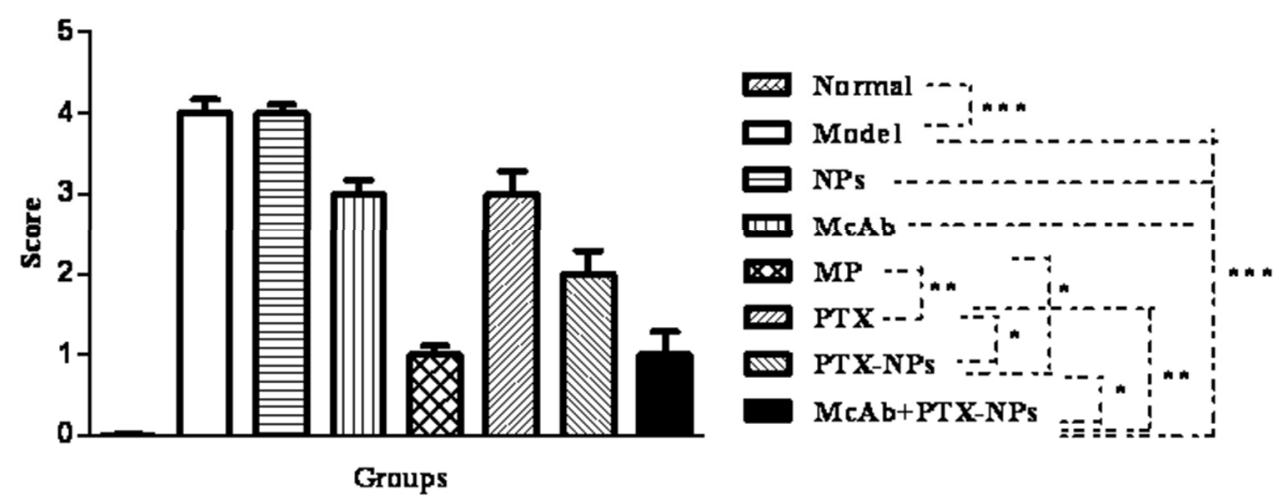

B

Figure 6: Histological analysis of kidneys of MM mice. A. Tissue sections derived from MM mice 4 weeks after treatments with different agents were stained with either masson (M) or PAS (P) Arrows indicate representative damaged areas as described in the text. B. Quantitative analysis of renal damages in treated mice. ${ }^{*} p<0.05,{ }^{* *} p<0.01$ and ${ }^{* * *} p<0.001$.

\section{Isolation of MM CD138- ${ }^{-} \mathrm{CD34}^{-}$cells}

CD138 ${ }^{-}$cells were isolated from human MM RPMI 8226 cells or BMMCs from MM patients using mouse anti-human $\mathrm{CD} 138$ and anti-human CD34 microbeads (Miltenyi Biotec, Germany) followed by immune magnetic activated cell sorting (Miltenyi Biotec, Germany) as described previously [23-25, 28, 29], and the positive rate of $\mathrm{CD} 138^{-} \mathrm{CD} 34^{-}$cells checked by FACS was about 95\% (data not shown).

\section{Establishment of a MM xenograft model}

NOD/SCID mice were injected with $1 \times 10^{7} \mathrm{MM}$ CD138-CD34- cells through tail veins. Eighteen days after injection, urine protein was collected by using a mouse metabolic cage and measured by enzymelinked immunosorbent assay (ELISA). Micro-computer tomography (Micro-CT) scanning and ultrasonography were used to examine bone lesions and renal damages in mice. MM-bearing mice were randomly divided into eight groups of equal size (3 mice), including model group (mice treated with PBS), NPs group (treated with 1.2 $\mathrm{mg} / \mathrm{kg} \mathrm{Fe} \mathrm{O}_{4} \mathrm{NPs}$ ), $\mathrm{McAb}$ group (treated with $0.5 \mathrm{mg} / \mathrm{kg}$ anti-ABCG2 McAb), PTX group (treated with $4 \mathrm{mg} /$ $\mathrm{kg}$ PTX), PTX-NPs group (treated with $0.122 \mathrm{mg} / \mathrm{kg}$ PTX+1.2 mg/kg NPs), McAb-PTX-NPs group (treated with $0.5 \mathrm{mg} / \mathrm{kg}$ anti-ABCG2 $\mathrm{McAb}$ and $0.122 \mathrm{mg} / \mathrm{kg}$ PTX+1.2 mg/kg NPs), MP group (treated with melphalan $7.5 \mathrm{mg} / \mathrm{kg}$ and prednisone $3 \mathrm{mg} / \mathrm{kg}$ ) as the positive control, and normal group (no treatment). To test the antitumor effect of agents, $200 \mu \mathrm{L}$ of each was intravenously injected into MM-bearing mice once a week with total of four times 18 days after the mice were injected with CSCs $[1,29-31]$. To analyze tumorigenicity, NOD/ SCID mice were subcutaneously injected with $5 \times 10^{4}$ $\mathrm{CD} 138^{-} \mathrm{CD} 34^{-}$cells derived from the bone marrow of MM patients, and tumors palpable at the injection sites were examined after 14 days. All the animal experiments were repeated at least twice.

\section{Preparation of PTX-NPs}

Oleic acid-coated iron oxide $\mathrm{NPs}\left(\mathrm{Fe}_{3} \mathrm{O}_{4} @ \mathrm{OA}\right.$ NPs) were synthesized by a two-step method to produce PTX-NPs as described previously [32]. Briefly, $\mathrm{Fe}_{3} \mathrm{O}_{4} @$ OA NPs were suspended in tetrahydrofuran at ratio of 1:5 (w/w). The mixture was slowly added into and $1 \%$ polyoxyethylene/polyoxypropylene copolymer solution under sonication and the resulting suspension was stirred to evaporate tetrahydrofuran, and was filtered and lyophilized. The drug is loaded on PTX-NPs at a ratio of $10.71 \%(\mathrm{PTX} / \mathrm{Fe}$, wt). The morphology of PTX-NPs after rehydration was observed by TEM 
A
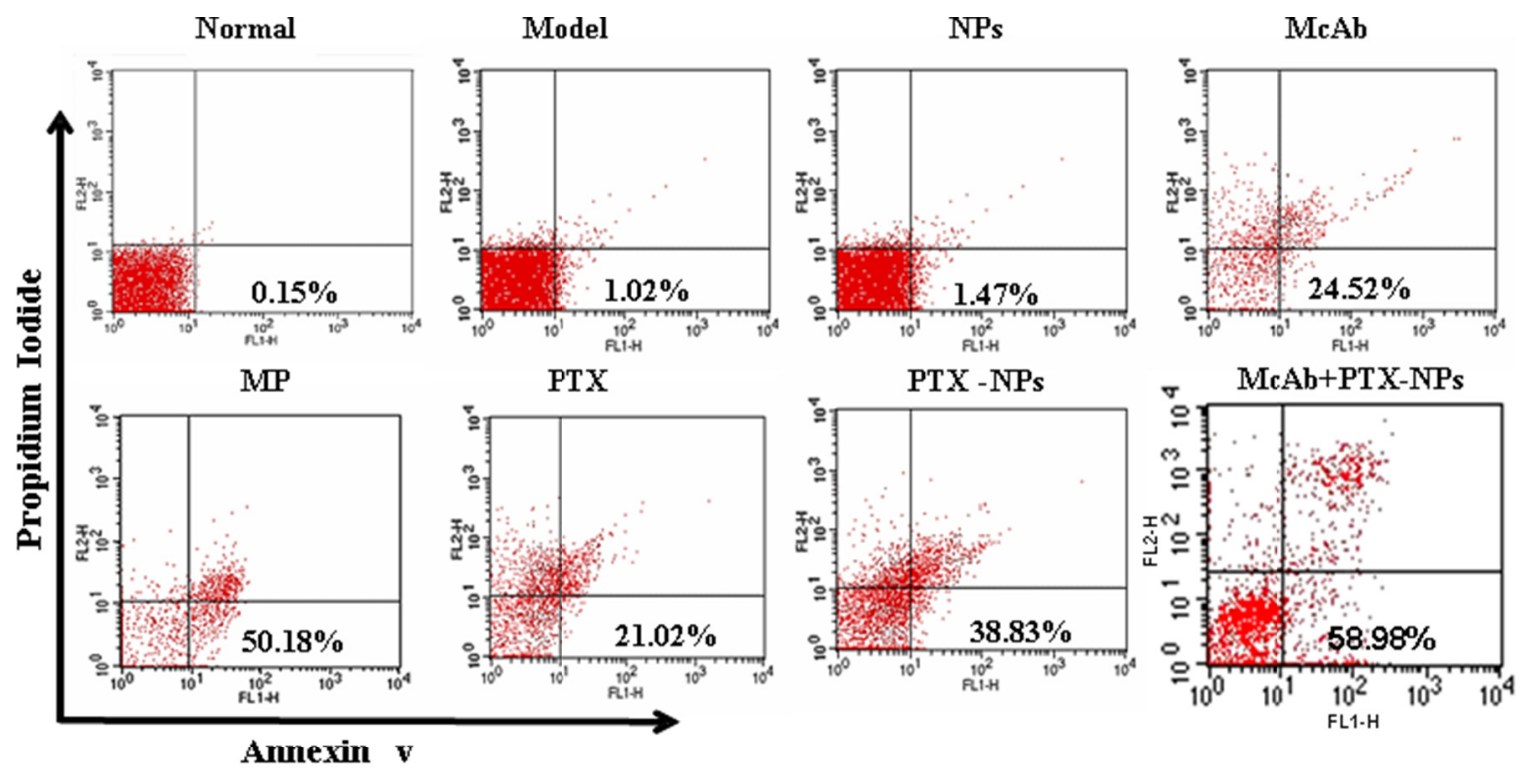

B
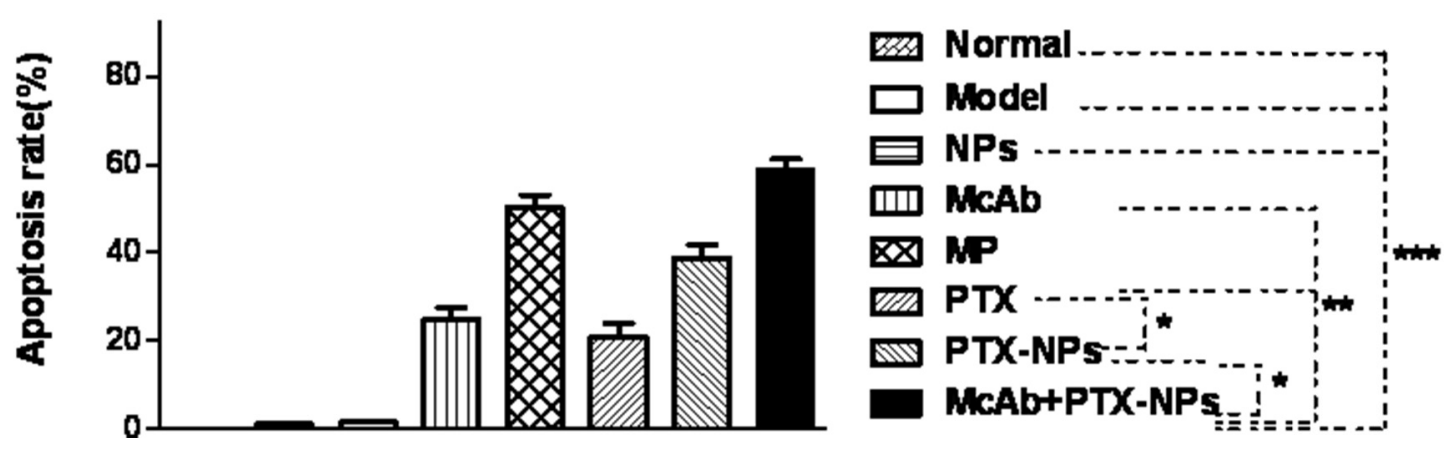

Figure 7: Strong apoptosis induced by McAb+PTX-NPs in BMMCs isolated from MM mice. A. Apoptosis of BMMCs derived from treated MM mice was analyzed as described in the Method. B. Quantification of apoptotic BMMCs. ${ }^{*} p<0.05, * * p<0.01$ and $* * * p<0.001$.

using microscope JEM-2000EX (JEOL Ltd., Japan). The hydrodynamic diameter, size distribution and zeta potential of PTX-NPs were examined by DLS with Malvern Zetasizer 3000 (Malvern Instruments Co., H.K.).

\section{Analysis of bone mineral density and peak systolic blood flow velocities of renal artery}

The bone mineral density (BMD) of the humerus and femurs in mice was measured by in vivo Micro-CT imaging with MCT-1108 (China) at a setting of voltage $45 \mathrm{kV}$ and electric current $80 \mu \mathrm{A}[33,34]$. The peak systolic BFV of renal artery was detected by ultrasonography with Vevo 2100 (Visualsonic, Germany). All the measurements were performed once a week with total of four times [35].

\section{Histological analysis of lytic bone lesions and renal impairments}

After four weeks of treatment, mice were anesthetized with phenobarbital sodium and sacrificed by cervical dislocation. The humerus and femurs as well as the kidneys were harvested, and next steps were performed as described previously $[1,13]$.

\section{Measurement of serum FLC, calcium, IL-6 and urine protein and red blood cells}

Serum free light chains (FLCs), calcium, cytokine IL-6 and urine protein were measured by ELISA-based kits according to the manufacturer's protocol (eBioscience company, USA) [17, 35]. Red blood cells (RBCs) were counted as described previously [18]. 


\section{Analysis of BMMC apoptosis}

$5 \times 10^{5}$ BMMCs isolated from the MM bearing mice were seeded into a 96-well plate $(100 \mu \mathrm{L} /$ well $)$, stained with FITC-conjugated Annexin V and Propidium Iodide (PI), and resuspended in PBS. The suspented cells at $100 \mu \mathrm{L}$ were incubated with $5 \mu \mathrm{L}$ Annexin V-FITC (KeyGen Biotech. Co. Ltd) and $10 \mu \mathrm{L}$ of $50 \mu \mathrm{g} / \mathrm{mL}$ PI for $15 \mathrm{~min}$ at room temperature in the dark. The stained cells were analyzed within 1 hour by flow cytometry using FACS Caliber (BD, U.S.A) [19].

\section{Statistical analyses}

The data were plotted as mean \pm SD and analyzed for statistical significance by two-tailed paired Student's $t$ test or repeated measures analysis of variance (ANOVA). $P$ values less than 0.05 were considered statistically significant. Analyses were performed with the Graph Pad Prism 3.0 statistical software package (Graph Pad Company, USA).

\section{ACKNOWLEDGMENTS AND FUNDING}

This work was supported by the 973 National Nature Science Foundation of People's Republic of China (2011CB933500) and Graduate Student Research and Innovation Program of Jiangsu Province (CXZZ-0174) as well as the National Natural Science Foundation of China (No. 81001412, 61127002 and 61179035).

\section{CONFLICTS OF INTEREST}

The authors declared that they have no conflict of interest.

\section{REFERENCES}

1. Hideshima T, Bergsagel PL, Kuehl WM, Anderson KC. Advances in biology of multiple myeloma: clinical applications. Blood. 2004; 104:607-618.

2. Matsui W, Wang Q, Barber JP, Brennan S, Smith BD, Borrello I, McNiece I, Lin L, Ambinder RF, Peacock C, Watkins DN, Huff CA, Jones RJ. Clonogenic multiple myeloma progenitors, stem cell properties, and drug resistance. Cancer Res. 2008; 68:190-197.

3. Olechnowicz SW, Edwards CM. Contributions of the host microenvironment to cancer-induced bone disease. Cancer Res. 2014; 74:1625-1631.

4. Mitsiades CS, Mitsiades NS, Richardson PG, Munshi NC, Anderson KC. Multiple myeloma: a prototypic disease model for the characterization and therapeutic targeting of interactions between tumor cells and their local microenvironment. J Cell Biochem. 2007; 101:950-968.
5. Abe M, Harada T, Matsumoto T. Concise review: Defining and targeting myeloma stem cell-like cells. Stem Cells. 2014; 32:1067-1073.

6. Drain S, Flannely L, Drake MB, Kettle P, Orr N, Bjourson AJ, Catherwood MA, Alexander HD. Multidrug resistance gene expression and $\mathrm{ABCB} 1 \mathrm{SNPs}$ in plasma cell myeloma. Leuk Res. 2011; 35:1457-1463.

7. Tan KW, Li Y, Paxton JW, Scheepens A. Identification of novel dietary phytochemicals inhibiting the efflux transporter breast cancer resistance protein (BCRP/ABCG2). Food Chem. 2013; 138:2267-2274.

8. Dou J, Jiang C, Wang J, Zhang X, Zhao F, Hu W, He X, Li X, Zou D, Gu N. Using ABCG2-Molecule- Expressing Side Population Cells to Identity Cancer Stem-like Cells in a Human Ovarian cell line. Cell Biol Int. 2011; 35:227-234.

9. Nooter K, Stoter G. Molecular mechanisms of multidrug resistance in cancer chemotherapy. Pathol Res Pract. 1996; 192:768-780.

10. Yang F, Li Y, Chen Z, Zhang Y, Wu J, Gu N. Superparamagnetic iron oxide nanoparticle-embedded encapsulated microbubbles as dual contrast agents of magnetic resonance and ultrasound imaging. Biomaterials. 2009; 30:3882-3890.

11. Xie H, Zhu Y, Jiang W, Zhou Q, Yang H, Gu N, Zhang Y, $\mathrm{Xu} \mathrm{H}, \mathrm{Xu} \mathrm{H}$, Yang X. Lactoferrin-conjugated superparamagnetic iron oxide nanoparticles as a specific MRI contrast agent for detection of brain glioma in vivo. Biomaterials. 2011; 32:495-502.

12. Hu K, Dou J, Yu F, He X, Yuan X, Wang Y, Liu C, Gu N. An ocular mucosal administration of nanoparticles containing DNA vaccine $\mathrm{pRSC}$-gD-IL-21 confers protection against mucosal challenge with herpes simplex virus type 1 in mice. Vaccine. 2011; 29:1455-1462.

13. Yu F, Wang J, Dou J, Yang H, Wang Y, Xu W, Zhang Y, $\mathrm{Hu} \mathrm{K}, \mathrm{Gu}$ N. Nanoparticle-based adjuvant for enhanced protective efficacy of DNA vaccine Ag85A-ESAT6-IL-21 against Mycobacterium tuberculosis infection. Nanomedicine. 2012; 8:1337-1344.

14. Palombo M, Deshmukh M, Myers D, Gao J, Szekely Z, Sinko PJ. Pharmaceutical and toxicological properties of engineered nanomaterials for drug delivery. Annu Rev Pharmacol Toxicol. 2014; 54:581-598.

15. Feng SS. Chemotherapeutic Engineering: Concept, Feasibility, Safety and Prospect-A Tribute to Shu Chien's 80th Birthday. Cell Mol Bioengineering. 2011; 4:708-716.

16. Taiani JT, Buie HR, Campbell GM, Manske SL, Krawetz RJ, Rancourt DE. Embryonic stem cell therapy improves bone quality in a model of impaired fracture healing in the mouse tracked temporally using in vivo micro-CT. Bone. 2014; 64:263-272.

17. Kolosenko I, Grandér D, Tamm KP. IL-6 Activated JAK/ STAT3 Pathway and Sensitivity to Hsp90 Inhibitors in Multiple Myeloma. Curr Med Chem. 2014; 21:3042-3047. 
18. Xiang D, Cong Y, Wang C, Yue J, Ma X, Lu Y, Liu P, Ma J. Development of microscopic review criteria by comparison urine flow cytometer, strip and manual microscopic examination. Clin Lab. 2012; 58:979-985.

19. Saini V, Shoemaker RH. Potential for therapeutic targeting of tumor stem cells. Cancer Sci. 2010; 101:16-21.

20. Cirstea D, Hideshima T, Rodig S. Dual Inhibition of Akt/ Mammalian Target of Rapamycin Pathway by Nanoparticle Albumin-Bound-Rapamycin and Perifosine Induces Antitumor Activity in Multiple Myeloma. Mol Cancer Ther. 2010; 9:963-975.

21. Raaijmakers MH, de Grouw EP, Heuver LH, van der Reijden BA, Jansen JH, Scheffer G. Impaired breast cancer resistance protein mediated drug transport in plasma cells in multiple myeloma. Leuk Res. 2005; 29:1455-1458.

22. Turner JG, Gump JL, Zhang C, Cook JM, Marchion D, Hazlehurst L, Munster P, Schell MJ, Dalton WS, Sullivan DM. ABCG2 expression, function, and promoter methylation in human multiple myeloma. Blood. 2006; 108:3881-3889.

23. Yang C, Xiong F, Wang J, Dou J, Chen J, Chen D, Zhang Y, Luo S, Gu N. Anti-ABCG2 monoclonal antibody in combination with paclitaxel nanoparticles against cancer stem-like cell activity in multiple myeloma. Nanomedicine (Lond). 2014; 9:45-60.

24. Yang C, He X, Song L, Zhan X, Zhang Y, Dou J, Gu N. $\gamma$-Fe2O3 Nanoparticles Increase the Therapeutic Efficacy of Combination with Paclitaxel and Anti-ABCG2 Monoclonal Antibody on Multiple Myeloma Cancer Stem Cells in Mouse Model. J Biomed Nanotechnol. 2014; 10:336-344.

25. Matsui W, Huff CA, Wang Q, Malehorn MT, Barber J, Tanhehco Y, Smith BD, Civin CI, Jones RJ. Characterization of clonogenic multiple myeloma cells. Blood. 2004; 103:2332-2336.

26. Bhutani M, Landgren O. Imaging in smoldering (asymptomatic) multiple myeloma: Past, present and future. Radiologe. 2014; 54:572-581.
27. Abedi M, Elfenbein GJ. Novel approaches in the treatment of multiple myeloma. Med Health RI. 2003; 86:231-235.

28. Dou J, Pan M, Wen P, Li Y, Tang Q, Chu L. Isolation and Identification of Cancer Stem Like Cells fromMurine Melanoma Cell Lines. Cell Mol Immunol. 2007; 4:467-472.

29. Chen D, Zhang Y, Wang J, Chen J, Yang C, Cai K. MicroRNA-200c overexpression inhibits tumorigenicity and metastasis of $\mathrm{CD} 117^{+} \mathrm{CD} 44^{+}$ovarian cancer stem cells by regulating epithelial- mesenchymal transition. J Ovarian Res. 2013; 6:50.

30. Chalaris A, Schmidt-Arras D, Yamamoto K, Rose-John S. Interleukin-6 trans-signaling and colonic cancer associated with inflammatory bowel disease. Dig Dis. 2012; 30:492-499.

31. Cea M, Cagnetta A, Fulciniti M, Rose-John S. Targeting NAD+salvage pathway induces autophagy in multiple myeloma cells via mTORC1 and extracellular signalregulated kinase (ERK1/2) inhibition. Blood. 2012; 120:3519-3529.

32. Guan N, Liu C, Sun D. A facile method to synthesize carboxyl-functionalized magnetic polystyrene nanospheres. Colloids and Surfaces A: Physicochem Eng Aspects. 2009; 335:174-180.

33. De Weerdt S. Animal models: Towards a myeloma mouse. Nature. 2011; 480:S38-S39.

34. Bonnin P, Sabaa N, Flamant M, Debbabi H, Tharaux PL. Ultrasound imaging of renal vaso-occlusive events in transgenic sickle mice exposed to hypoxic stress. Ultrasound Med Biol. 2008; 34:1076-1084.

35. Brioli A, Giles H, Pawlyn C, Campbell JP, Kaiser MF, Melchor L, Jackson GH, Gregory WM, Owen RG, Child JA, Davies FE, Cavo M, Drayson MT, Morgan GJ. Serum free light chain evaluation as a marker for the impact of intra-clonal heterogeneity on the progression and treatment resistance in multiple myeloma. Blood. 2014; 123:3414-3419. 